Pure and Applied Mathematics Quarterly

Volume 3, Number 3

(Special Issue: In honor of

Leon Simon, Part 2 of 2$)$

$801-825,2007$

\title{
Regularity of Minimizers for Three Elliptic Problems: Minimal Cones, Harmonic Maps, and Semilinear Equations
}

\author{
Xavier Cabré and Antonio Capella
}

\begin{abstract}
We discuss regularity issues for minimizers of three nonlinear elliptic problems. They concern minimal cones, minimizing harmonic maps into a hemisphere, and radial local minimizers of semilinear elliptic equations. We describe the strong analogies among the three regularity theories. They all use a method originated in a paper of J. Simons on the area minimizing properties of cones.
\end{abstract}

\section{INTRODUCTION}

In this article we describe regularity results for three different nonlinear elliptic problems. They concern minimal cones, minimizing harmonic maps into a hemisphere, and radial local minimizers of semilinear elliptic equations. The three statements on regularity, their optimality, and also the methods used in their proofs, share very strong analogies.

The three proofs use a method based on the (semi-)stability property of minimizers (that is, on the nonnegativeness of the second variation of energy at a minimizer). The method originated in the paper of J. Simons [21] on the area minimizing properties of cones. We emphasize that the regularity results that we describe hold for minimizers, but not for general solutions.

Received February 6, 2006. 
Another analogy among the three problems is that the regularity of minimizers holds up to a certain space dimension which, in addition, is known to be optimal. Its optimality is shown by exhibiting an explicit example of singular minimizer in higher dimensions.

In addition, we will see that for each of the problems, the regularity result in bounded domains is related to a corresponding Liouville type theorem for minimizers in the whole space.

Section 2 describes Simons [21] result on the regularity of minimal cones up to dimension 7, as well as Bombieri-De Giorgi-Giusti [2] result on the minimality of the Simons cone in higher dimensions. These works date from the end of the sixties. Simons result is the crucial ingredient to establish the regularity, indeed analyticity, of minimal sets in low space dimensions.

Section 3 describes similar results for minimizing harmonic maps from $\mathbb{R}^{n}$ into the closed upper hemisphere $\overline{S_{+}^{N}}$ of the unit sphere $S^{N} \subset \mathbb{R}^{N+1}$. Here the optimal result states that regularity holds whenever $n \leq 6$. These works date from the early eighties, and are due to Jäger-Kaul [15], Giaquinta-Souček [10], and Schoen-Uhlenbeck [20].

In the last section we present very recent analogue developments due to the authors $[4,5]$ for radial local minimizers of semilinear elliptic equations. Our results establish the boundedness, and hence regularity, of radial local minimizers in a ball up to dimension 9. This result holds for all locally Lipschitz reaction nonlinearities. In addition, the limiting dimension 9 is shown to be optimal.

We also discuss the results of [4], where we use similar methods to prove certain Liouville type results for local minimizers. They state that, up to dimension 10, every semi-stable bounded radial solution in the whole space must be constant.

There is a well known connection between certain semilinear elliptic equations and minimal surfaces theory. Namely, phase transitions or interfaces may be modeled by semilinear equations. They appear in physical problems when two different states coexist and there is a balance between two opposite tendencies: a diffusive effect that tends to mix the materials and a reaction mechanism that drives them into their pure state. Due to surface tension, interfaces tend to minimize their area as the reaction term becomes stronger. A precise variational 
justification of this is given by the classical Modica-Mortola $\Gamma$-convergence result (see [1]).

The classical result on the flatness of minimal graphs in low dimensions (see [13]) has a semilinear analogue. It is a conjecture posed by E. De Giorgi in 1978 and for which progress has been made only recently. Here the main results state that bounded solutions in the whole space which are monotone in one variable are always local minimizers of the energy, and that in low space dimensions they are necessarily functions of only one Euclidean variable (1d symmetry). Recently the conjecture has been explored quite intensively by various authors (see $[1,16,19]$ for more information). Our work on the conjecture is precisely what led us to the semilinear regularity results for minimizers described in this paper.

\section{Minimal cones}

In this section we discuss classical results on minimal surfaces theory concerned with the regularity of minimal cones. As we will see, by a blow-up method the existence or not of singular minimal cones will be the key point on the study of the regularity of general minimal surfaces.

A cone $E \subset \mathbb{R}^{n}$ is a set of the form $E=\{t y: t>0, y \in \Sigma\}$, where $\Sigma \subset S^{n-1}$ is a given subset of the unit sphere $S^{n-1} \subset \mathbb{R}^{n}$. We take $\Sigma$ open and regular, so that $\partial E \backslash\{0\}$ is a regular hypersurface in $\mathbb{R}^{n}$.

The next theorem is due to Simons (see Theorem 10.10 of [13]).

Theorem 2.1 (Simons [21]). Let $E \subset \mathbb{R}^{n}$ be a cone such that $\partial E \backslash\{0\}$ is regular and has zero mean curvature. Assume that the second variation of area at $\partial E \backslash\{0\}$ is nonnegative, that is,

$$
\int_{\partial E}\left\{|\delta \xi|^{2}-c^{2} \xi^{2}\right\} d H_{n-1} \geq 0
$$

for every $\xi \in C^{1}(\partial E)$ with compact support outside the origin.

If $n \leq 7$, then $\partial E$ is a hyperplane.

From this result, it follows that no singular minimal cone exists in $\mathbb{R}^{n}$ for $n \leq 7$. Shortly after [21], Bombieri, De Giorgi, and Giusti proved that this result is the best possible. 
Theorem 2.2 (Bombieri-De Giorgi-Giusti [2]). The Simons cone

$$
S=\left\{x \in \mathbb{R}^{2 m}: x_{1}^{2}+\ldots+x_{m}^{2}>x_{m+1}^{2}+\ldots+x_{2 m}^{2}\right\}
$$

has least perimeter in $\mathbb{R}^{2 m}$ if and only if $2 m \geq 8$.

The rest of this section is dedicated to explain the proofs and importance of these two results. In particular, we will explain the meaning of the quantities appearing in (2.1). Note that, in the second theorem, the necessity of condition $2 m \geq 8$ for minimality follows automatically from Theorem 2.1. Hence, the point of Theorem 2.2 is the sufficiency of condition $2 m \geq 8$. Note also that the boundary of the Simons cone has zero mean curvature everywhere away from the origin in every dimension $2 m$.

Note also that we are using the term "minimal" in the variational sense, as in De Giorgi's theory of minimal surfaces that we describe next. Recall that in certain literature, "minimal surface" means a surface with zero mean curvature, while "stable minimal surface" means a surface with zero mean curvature and satisfying (2.1), that is, with nonnegative second variation of area. Again, we do not use here this terminology.

In De Giorgi's theory of minimal surfaces (see the book of Giusti [13] for a great exposition), one regards a hypersurface (or variety of dimension $n-1$ ) with finite area as the boundary of a Borel set $E \subset \mathbb{R}^{n}$ with locally finite perimeter. To be more precise, if $\varphi_{E}$ is the characteristic function of the set $E$ then the perimeter of $E$ in an open set $\Omega \subset \mathbb{R}^{n}$ is given by

$$
P(E, \Omega)=\int_{\Omega}\left|D \varphi_{E}\right|=\sup \left\{\int_{E} \operatorname{div} g d x: g \in C_{0}^{1}\left(\Omega, \mathbb{R}^{n}\right),\|g\|_{\infty} \leq 1\right\} .
$$

A minimal surface in $\Omega$ is meant to be the part in $\Omega$ of the boundary of a set $E \subset \mathbb{R}^{n}$ with minimal perimeter in $\Omega$ (called a minimal set in $\Omega$ ). That is, the set $E \subset \mathbb{R}^{n}$ must satisfy $P(E, \Omega) \leq P(F, \Omega)$ for all $F \subset \mathbb{R}^{n}$ such that $(E \backslash F) \cup(F \backslash E)$ has compact closure contained in $\Omega$.

Next, one considers local perturbations of a set $E$ and compute the first and second variations of the perimeter or area functional. To this end, assume that $E \subset \mathbb{R}^{n}$ has finite perimeter in $B_{1}$, and let $\left\{F_{t}\right\}$ be a one-parameter family of diffeomorphisms $F_{t}: \mathbb{R}^{n} \rightarrow \mathbb{R}^{n}$ such that $F_{0}$ is the identity and the maps $F_{t}-I$ have uniform compact support contained in $B_{1}$. Consider the sets $E_{t}=F_{t}(E)$. 
We are interested in the area functional

$$
A(t):=\int_{B_{1}}\left|D \varphi_{E_{t}}\right| .
$$

One proceeds by choosing a deformation $F_{t}=I+t \xi \nu$ (where $I$ is the identity) that shifts the original set in the normal direction $\nu$ to its boundary (here $\xi$ is a scalar function with compact support in $B_{1}$ ). It can be proved (see chapter 10 of [13]) that $A^{\prime}(0)$ and $A^{\prime \prime}(0)$ are given by

$$
\begin{aligned}
\left.\frac{d}{d t} \int_{B_{1}}\left|D \varphi_{E_{t}}\right|\right|_{t=0} & =\int_{\partial E} \mathcal{H} \xi d H_{n-1}, \\
\left.\frac{d^{2}}{d t^{2}} \int_{B_{1}}\left|D \varphi_{E_{t}}\right|\right|_{t=0} & =\int_{\partial E}\left\{|\delta \xi|^{2}-\left(c^{2}-\mathcal{H}^{2}\right) \xi^{2}\right\} d H_{n-1},
\end{aligned}
$$

where $\mathcal{H}=\mathcal{H}(x)$ is the mean curvature of $\partial E$ at $x, c^{2}=c^{2}(x)$ is the sum of the squares of the $n-1$ principal curvatures of $\partial E$ at $x, H_{n-1}$ denotes the $(n-1)$ dimensional Hausdorff measure, and the tangential derivative $\delta$ is defined as

$$
\delta_{i}=\partial_{x_{i}}-\nu_{i} \sum_{j=1}^{n} \nu_{j} \partial_{x_{j}},
$$

where again $\nu$ is the normal vector to $\partial E$ at $x$. In this context, the LaplaceBeltrami operator on $\partial E$ is defined as

$$
\mathcal{D}=\sum_{i=1}^{n} \delta_{i} \delta_{i} .
$$

If $E$ is a minimal set in $B_{1}$ then, since $E_{t}=F_{t}(E)$ coincide with $E$ outside $B_{1}$, we have

$$
\int_{B_{1}}\left|D \varphi_{E}\right| \leq \int_{B_{1}}\left|D \varphi_{E_{t}}\right|
$$

That is, the function $A(t)=\int_{B_{1}}\left|D \varphi_{E_{t}}\right|$ has a global minimum at $t=0$ and therefore, assuming appropriate smoothness, $A^{\prime}(0)=0$ and $A^{\prime \prime}(0) \geq 0$. By expression (2.2) it follows that $\partial E$ has zero mean curvature. This combined with (2.3) gives that the second variation of area at $\partial E$ being nonnegative is equivalent to condition (2.1) in Theorem 2.1.

Simons result (Theorem 2.1) on minimal cones leads to the following important regularity result for general minimal sets.

Theorem 2.3. If $n \leq 7$ and $E$ is a minimal set in a ball $B_{r} \subset \mathbb{R}^{n}$, then $\partial E \cap B_{r}$ is an analytic hypersurface. 
This result is Theorem 10.11 in [13], where a detailed proof can be found. The idea is to construct minimal cones as follows. If $E$ is a minimal set in $B_{r}$ and $0 \in \partial E$ is a possible singular point, we blow up the set $E$ around 0 . That is, we consider the sets

$$
E_{k}=\left\{x \in \mathbb{R}^{n}: \frac{x}{k} \in E\right\}, \quad k=1,2, \ldots
$$

By geometric invariance all $E_{k}$ are minimal sets and, up to subsequences, converge to a minimal set $\tilde{E}$ which, in addition, is a cone tangent to $E$ at 0 . Here a crucial monotonicity formula for the area of minimal surfaces is used. The set $E$ will be regular near the origin if and only if $\partial \tilde{E}$ is a hyperplane. Thus, the non existence of singularities of $\partial E$ is reduced to the non existence of singular minimal cones.

We turn now to the proof of Simons result on minimal cones, Theorem 2.1. It uses an inequality for the Laplacian on $\partial E$ of $c^{2}$ (recall that $c^{2}$ is the sum of the squares of the principal curvatures of $\partial E$ ), in the case when $\partial E$ is a stationary cone. Stationary means that the first variation of area equals zero. By (2.2) this is equivalent to $\mathcal{H}=0$.

Lemma 2.4. Let $E \subset \mathbb{R}^{n}$ be a cone such that $\partial E \backslash\{0\}$ is regular and has zero mean curvature. Then $c^{2}$ is homogeneous of degree -2 , and in $\partial E \backslash\{0\}$ we have

$$
\frac{1}{2} \mathcal{D} c^{2} \geq-c^{4}+|\delta c|^{2}+\frac{2 c^{2}}{|x|^{2}}
$$

This result is Lemma 10.9 of [13], where a proof can be found. We now use it to give the proof of Simons result.

Proof of Theorem 2.1. The key idea is to take $\xi=c \eta$ in (2.1), where $\eta$ is still arbitrary (with compact support outside the origin) and will be chosen later. We obtain

$$
\begin{aligned}
\int_{\partial E} c^{4} \eta^{2} d H_{n-1} & \leq \int_{\partial E}\left\{c^{2}|\delta \eta|^{2}+\eta^{2}|\delta c|^{2}+\frac{1}{2} \delta c^{2} \cdot \delta \eta^{2}\right\} d H_{n-1} \\
& =\int_{\partial E}\left\{c^{2}|\delta \eta|^{2}+\eta^{2}|\delta c|^{2}-\frac{1}{2} \eta^{2} \mathcal{D} c^{2}\right\} d H_{n-1}
\end{aligned}
$$

where we have used integration by parts. Now using Lemma 2.4 we obtain

$$
0 \leq \int_{\partial E} c^{2}\left\{|\delta \eta|^{2}-\frac{2 \eta^{2}}{|x|^{2}}\right\} d H_{n-1}
$$

for every $\eta \in C^{1}(\partial E)$ with compact support outside the origin. 
Obviously (2.4) will hold by approximation for every function $\eta$ provided that

$$
\int_{\partial E} c^{2} \frac{\eta^{2}}{|x|^{2}} d H_{n-1}<\infty
$$

By Lemma 2.4, $c^{2}$ is homogeneous of degree -2 . Thus (2.4) holds for every $\eta$ such that

$$
\int_{\partial E} \eta^{2}|x|^{-4} d H_{n-1}<\infty
$$

If $r_{1}=\max \{|x|, 1\}$ and $r=|x|$, we now choose

$$
\eta=r^{\alpha} r_{1}^{\beta}
$$

Then

$$
\int_{\partial E} \eta^{2}|x|^{-4} d H_{n-1}=H_{n-2}\left(\partial E \cap \partial B_{1}\right) \int_{0}^{\infty} r^{2 \alpha} r_{1}^{2 \beta} r^{n-6} d r
$$

Thus, to make the integral finite we must have $2 \alpha+n-6>-1$ and at the same time $2(\alpha+\beta)+n-6<-1$. That is

$$
\alpha>\frac{5-n}{2} \text { and } \quad \alpha+\beta<\frac{5-n}{2} .
$$

If inequalities (2.5) hold, then (2.4) becomes

$$
0 \leq\left(\alpha^{2}-2\right) \int_{\partial E \cap B_{1}} r^{2 \alpha-2} c^{2} d H_{n-1}+\left[(\alpha+\beta)^{2}-2\right] \int_{\partial E \backslash B_{1}} r^{2(\alpha+\beta)-2} c^{2} d H_{n-1} .
$$

If $3 \leq n \leq 7$ (the case $n=2$ is treated separately) then $(5-n)^{2} / 4<2$, and we can choose $\alpha$ and $\beta$ satisfying (2.5) and such that $\alpha^{2}<2$ and $(\alpha+\beta)^{2}<2$. It then follows that $c^{2} \equiv 0$, and hence $\partial E$ is a hyperplane.

We finish this section giving a clever proof due to U. Massari and M. Miranda of the minimality of the Simons cone in dimension $2 m \geq 8$. Recall that this is Theorem 2.2 above (and Theorem 16.4 in [13]).

Proof of Theorem 2.2. Recall that the area functional for graphs $v: \Omega \subset \mathbb{R}^{2 m} \rightarrow \mathbb{R}$ is given by

$$
\mathcal{A}(v, \Omega)=\int_{\Omega} \sqrt{1+|D v|^{2}} d x
$$

where $\Omega \subset \mathbb{R}^{2 m}$ is a bounded set. Its Euler-Lagrange equation is

$$
\mathcal{E}(v):=\left(1+|D v|^{2}\right) \Delta v-v_{x_{i} x_{j}} v_{x_{i}} v_{x_{j}}=0,
$$

where $\mathcal{E}$ is the minimal graph operator, and summation over repeated indexes is understood. 
For $x \in \mathbb{R}^{2 m}$, we set $y=\left(x_{1}, \ldots, x_{m}\right)$ and $z=\left(x_{m+1}, \ldots, x_{2 m}\right)$. Consider the function

$$
v=\left(|y|^{2}-|z|^{2}\right)\left(|y|^{2}+|z|^{2}\right),
$$

for which expression (2.6) gives

$$
\mathcal{E}(v)=4\left(|y|^{2}-|z|^{2}\right)\left\{(m+2)\left[1+16\left(|y|^{6}+|z|^{6}\right)\right]-48\left(|y|^{2}+|z|^{2}\right)\left(|y|^{4}+|z|^{4}\right)\right\} .
$$

For $m \geq 4$ the quantity in brackets is positive, and therefore $v$ is a subsolution $(\mathcal{E}(v) \geq 0)$ in $S=\left\{|y|^{2}>|z|^{2}\right\}$ and a supersolution in $\mathbb{R}^{2 m} \backslash \bar{S}$. If we set for $k>0$,

$$
v_{k}(x)=\frac{1}{k} v(k x)=k^{3} v(x),
$$

then

$$
\mathcal{E}\left(v_{k}\right)(x)=k \mathcal{E}(v)(k x) .
$$

Therefore, $v_{k}$ is also a subsolution and a supersolution in $S$ and in $\mathbb{R}^{2 m} \backslash \bar{S}$, respectively.

Now we consider the Dirichlet problem for the area functional

$$
\mathcal{A}\left(u, B_{R}\right)=\int_{B_{R}} \sqrt{1+|D u|^{2}} d x
$$

in a ball $B_{R}$ of radius $R$, with boundary data $v_{k}$ on $\partial B_{R}$. This problem has a unique solution $u_{k}$ (see Theorem 12.10 in [13]). From uniqueness and the symmetry of $v_{k}$, we deduce that $u_{k}=0$ on $\partial S$ and therefore, by the maximum principle we conclude that $u_{k} \geq v_{k}$ in $S$ and $u_{k} \leq v_{k}$ in $B_{R} \backslash \bar{S}$.

Let now $k \rightarrow \infty$. The sequence $v_{k}$, and therefore also $u_{k}$, tends to $+\infty$ in $S$ and to $-\infty$ in $B_{R} \backslash \bar{S}$. By Proposition 16.5 in [13], the limit $u$ of $u_{k}$ given by

$$
u(x)=\left\{\begin{array}{l}
+\infty, x \in S \\
-\infty, x \in B_{R} \backslash \bar{S}
\end{array}\right.
$$

is a quasi-solution of the minimal graph equation. This means that its subgraph $U=\left\{(x, t) \in \mathbb{R}^{2 m} \times \mathbb{R}: t<u(x)\right\}=S \times \mathbb{R}$ locally minimizes the perimeter in $\mathbb{R}^{2 m} \times \mathbb{R}$, i.e.,

$$
\int_{\tilde{K}}\left|D \varphi_{U}\right| \leq \int_{\tilde{K}}\left|D \varphi_{V}\right|
$$

for every $V \subset \mathbb{R}^{2 m} \times \mathbb{R}$ coinciding with $U$ outside of some compact set $\tilde{K}$. As before, $\varphi_{U}$ and $\varphi_{V}$ are the characteristic functions of the sets $U$ and $V$, respectively. 
To show that $S$ has least perimeter we argue by contradiction. Assume that there exists a compact set $K \subset \mathbb{R}^{2 m}$, a positive number $\delta$, and a set $F$ coinciding with $S$ outside $K$, such that

$$
\int_{K}\left|D \varphi_{F}\right| \leq \int_{K}\left|D \varphi_{S}\right|-\delta
$$

For $T>0$ set

$$
F_{T}= \begin{cases}F \times \mathbb{R} & \text { in } K_{T}=K \times[-T, T] \\ S \times \mathbb{R}=U & \text { outside } K_{T} .\end{cases}
$$

We have

$$
\int_{K_{T}}\left|D \varphi_{F_{T}}\right| \leq \int_{K_{T}}\left|D \varphi_{F \times \mathbb{R}}\right|+2|K| \leq \int_{K_{T}}\left|D \varphi_{U}\right|-2 T \delta+2|K|<\int_{K_{T}}\left|D \varphi_{U}\right|
$$

whenever $T \delta>|K|$. But in this case $U$ would not be a local minimum of the area in $\mathbb{R}^{2 m} \times \mathbb{R}$, a contradiction.

Therefore $S$ has least perimeter and the proof is completed.

\section{Minimizing harmonic maps into a Closed hemisphere}

Consider the energy

$$
E(u)=\frac{1}{2} \int_{\Omega}|D u|^{2} d x
$$

for $H^{1}$ maps $u: \Omega \subset \mathbb{R}^{n} \rightarrow \mathcal{N}$ from a domain $\Omega$ of $\mathbb{R}^{n}$ into a Riemannian manifold $\mathcal{N}$ of dimension $N$. A critical point of $E$ is called a (weakly) harmonic map. When a map minimizes $E$ among all maps with same boundary values on $\partial \Omega$, then it is called a minimizing harmonic map.

Existence and regularity of harmonic maps has been widely studied under many different assumptions (see for instance $[9,14]$ and references within).

We focus our attention to the case of minimizing harmonic maps into the closed upper hemisphere

$$
\mathcal{N}=\overline{S_{+}^{N}}=\left\{y \in \mathbb{R}^{N+1}:|y|=1, y_{N+1} \geq 0\right\} .
$$

In this case, the equation for harmonic maps is given by

$$
-\Delta u=|D u|^{2} u \quad \text { in } \Omega \text {. }
$$

Giaquinta and Souček [10], and independently Schoen and Uhlenbeck [20], proved the following result. 
Theorem 3.1 (Giaquinta-Souček [10], Schoen-Uhlenbeck [20]). Let $u: B_{1} \subset \mathbb{R}^{n} \rightarrow \overline{S_{+}^{N}}$ be a minimizing harmonic map, homogeneous of degree zero, into the closed upper hemisphere $\overline{S_{+}^{N}}$. If $3 \leq n \leq 6$, then $u$ is constant.

The next explicit example shows that the previous result is optimal.

Theorem 3.2 (Jäger and Kaul [15]). The equator map

$$
u_{*}: B_{1} \subset \mathbb{R}^{n} \rightarrow \overline{S_{+}^{n}}, \quad x \mapsto(x /|x|, 0)
$$

is a minimizing harmonic map on the class

$$
\mathcal{C}=\left\{u \in H^{1}\left(B_{1} \subset \mathbb{R}^{n}, S^{n}\right): u=u_{*} \text { on } \partial B_{1}\right\}
$$

if and only if $n \geq 7$.

Most of this section is devoted to prove Theorem 3.1 following [10]. This theorem automatically gives one part of the statement of Theorem 3.2. Namely, that the equator map $u_{*}$ is not minimizing for $3 \leq n \leq 6$. Earlier, Jäger and Kaul [15] had proved that $u_{*}$ is, even more, unstable when $3 \leq n \leq 6$. We will not present the proof of minimality of the equator map for $n \geq 7$ (see also the original paper [15] for this).

As in the previous section where a result on minimal cones led to a regularity result for general minimal surfaces, in the present context Theorem 3.1 (combined again with a blow-up procedure and a crucial monotonicity formula) has the following important consequence.

Theorem 3.3 (Giaquinta-Souček [10], Schoen-Uhlenbeck [20]). Assume that $n \leq 6$ and that $u$ is a minimizing harmonic map from a domain $\Omega \subset \mathbb{R}^{n}$ into the closed upper hemisphere $\overline{S_{+}^{N}}$.

Then, $u$ is regular. Moreover, if $\Omega=\mathbb{R}^{n}$ then $u$ is constant.

After these results, there have been many others on harmonic maps into spheres - some of them stronger in the sense that the restriction of mapping into a hemisphere is removed (see [14] for a survey).

Let us finally mention that, for general Riemmanian manifolds $\mathcal{M}$ and $\mathcal{N}$, Schoen and Uhlenbeck (and Giaquinta and Giusti in a special case) proved that every minimizing harmonic map from $\mathcal{M}$ to $\mathcal{N}$ is regular except in a singular set of Hausdorff dimension $d \leq \operatorname{dim} \mathcal{M}-3$ (see $[9,14]$ ). 
Proof of Theorem 3.1. We introduce the coordinates induced by the stereographic projection (with respect to the south pole) $P$ from $S^{N} \subset \mathbb{R}^{N+1}$ to $\mathbb{R}^{N}$. For the new function $v=P \circ u: B_{1} \subset \mathbb{R}^{n} \rightarrow \mathbb{R}^{N}$, the energy (3.1) (up to a constant factor) is given by

$$
E(v):=\int_{B_{1}} \frac{|D v|^{2}}{\left(1+|v|^{2}\right)^{2}} d x .
$$

In addition, we have $|v| \leq 1$ since the image of $u$ is contained in the closed upper hemisphere.

Next we note that for every bounded $w \in H^{1}\left(B_{1}, \mathbb{R}^{N}\right)$, one can construct a new map $\tilde{w}$, with $|\tilde{w}| \leq 1$, defined by

$$
\tilde{w}:= \begin{cases}w & \text { if }|w| \leq 1 \\ \frac{w}{|w|^{2}} & \text { if }|w| \geq 1\end{cases}
$$

and such that $E(w)=E(\tilde{w})$. Modulo the stereographic projection, $\tilde{w}$ corresponds to the reflected map of $w$ through the equator, which has image in the closed upper hemisphere (and hence $|\tilde{w}| \leq 1$ ).

From this observation and the hypothesis that $v \in H^{1}\left(B_{1}, \mathbb{R}^{N}\right),|v| \leq 1$, minimizes the energy among all maps $w$ with $w=v$ on $\partial B_{1}$ and image in the closed upper hemisphere (i.e., $|w| \leq 1$ ), it follows that $v$ also minimizes the energy in the class

$$
\left\{w \in H^{1}\left(B_{1}, \mathbb{R}^{N}\right): w \text { bounded and } w=v \text { on } \partial B_{1}\right\} .
$$

In particular, $v$ is a solution of the Euler-Lagrange equation for (3.2):

$$
\delta E(v) \xi=2 \int_{B_{1}}\left\{\frac{D v \cdot D \xi}{\left(1+|v|^{2}\right)^{2}}-2 \frac{|D v|^{2}}{\left(1+|v|^{2}\right)^{2}} \frac{v \cdot \xi}{1+|v|^{2}}\right\} d x=0
$$

for every $\xi \in\left(H_{0}^{1} \cap L^{\infty}\right)\left(B_{1}, \mathbb{R}^{N}\right)$.

In (3.3) we choose

$$
\xi(x)=v(x) \eta(|x|),
$$

where $\eta$ is a smooth radial function with compact support in $B_{1}$. Using that $v$ is homogeneous of degree zero, we get

$$
\int_{\partial B_{1}} \frac{|D v|^{2}}{\left(1+|v|^{2}\right)^{2}} \frac{1-|v|^{2}}{1+|v|^{2}} d H_{n-1}=0 .
$$


Since $1-|v|^{2} \geq 0$, we deduce that either $v$ is constant (and then we are finished) or that

$$
|v| \equiv 1
$$

that we assume from now on.

From (3.3), we can compute the second variation of energy. Using that $|v| \equiv 1$, we obtain

$(3.4) \quad \delta^{2} E(v)(\xi, \xi)=\frac{1}{2} \int_{B_{1}}\left\{|D \xi|^{2}-4 v \cdot \xi D v \cdot D \xi-|D v|^{2}|\xi|^{2}+3(v \cdot \xi)^{2}|D v|^{2}\right\} d x \geq 0$

which is nonnegative since $v$ is a minimizer.

In the second variation (3.4), we insert

$$
\xi(x)=v(x)|D v(x)| \eta(|x|),
$$

where $\eta$ is a smooth radial function with compact support in $B_{1}$ (to be chosen later). We find

$$
\int_{B_{1}}\left\{c^{2}|D \eta|^{2}-\left[c^{4}+\frac{1}{2} \Delta c^{2}-|D c|^{2}\right] \eta^{2}\right\} d x \geq 0
$$

where

$$
c(x):=|D v(x)|
$$

We use now the following important inequality.

Lemma 3.4. Since $v$ is a harmonic map, homogeneous of degree zero, and with $|v| \equiv 1$, we have

$$
c^{4}+\frac{1}{2} \Delta c^{2}-|D c|^{2} \geq \frac{c^{2}}{|x|^{2}}+\frac{c^{4}}{n-1}
$$

where $c:=|D v|$.

This lemma is the analogue result of Lemma 2.4 for minimal cones. See [10] for a proof of the lemma, which also follows from Bochner identity (see [20]).

From (3.5) and Lemma 3.4 we deduce

$$
\int_{B_{1}}\left\{c^{2}|D \eta(|x|)|^{2}-\left[\frac{c^{2}}{|x|^{2}}+\frac{c^{4}}{n-1}\right] \eta^{2}(|x|)\right\} d x \geq 0,
$$

for every smooth radial function $\eta$ with compact support in $B_{1}$. 
Arguing by contradiction, we assume that $v$ is nonconstant, and hence $c^{2} \not \equiv 0$. Using in addition that $c^{2}$ is homogeneous of degree -2 , we obtain from the previous inequality that

$$
\int_{0}^{1} r^{n-3}\left(\eta^{\prime}\right)^{2} d r-\left(1+\frac{1}{n-1} \frac{\int_{\partial B_{1}} c^{4}}{\int_{\partial B_{1}} c^{2}}\right) \int_{0}^{1} r^{n-5} \eta^{2} d r \geq 0
$$

for every smooth $\eta$ with compact support in $[0,1)$.

Defining

$$
\sigma:=\frac{1}{n-1} \frac{\int_{\partial B_{1}} c^{4}}{\int_{\partial B_{1}} c^{2}}>0
$$

(3.6) combined with a simple approximation and rescaling argument, gives that

$$
\int_{0}^{\infty} r^{n-3}\left(\eta^{\prime}\right)^{2} d r-(1+\sigma) \int_{0}^{\infty} r^{n-5} \eta^{2} d r \geq 0
$$

for every function $\eta:(0,+\infty) \rightarrow \mathbb{R}$ for which the integrals in (3.7) converge. Now, choosing

$$
\eta(r):= \begin{cases}r^{\alpha} & \text { for } r \leq 1 \\ r^{\beta} & \text { for } r \geq 1\end{cases}
$$

with $\alpha:=(4-n+\varepsilon) / 2$ and $\beta:=(4-n-\varepsilon) / 2$ (where $\varepsilon>0)$, (3.7) leads to

$$
\frac{\alpha^{2}}{n-4+2 \alpha}-\frac{\beta^{2}}{n-4+2 \beta} \geq(1+\sigma)\left\{\frac{1}{n-4+2 \alpha}-\frac{1}{n-4+2 \beta}\right\} \text {. }
$$

Since $\varepsilon>0$ is arbitrary, we deduce that

$$
\frac{(4-n)^{2}}{2} \geq 2(1+\sigma)>2 .
$$

It follows that $|4-n|>2$, and hence $n>6$. The theorem is now proved.

\section{RADIAL LOCAL MINIMIZERS OF SEMILINEAR ELLIPTIC EQUATIONS}

In this section we present recent results of the authors $[5,4]$ on radial local minimizers of semilinear elliptic equations. As we will see, the results and their proofs have strong analogies with those already presented for minimal cones and minimizing harmonic maps.

Throughout this section, $G$ is a $C_{\text {loc }}^{1,1}(\mathbb{R})$ function (i.e., $G^{\prime}$ is locally Lipschitz). We write $g=G^{\prime}$. 
Consider the energy functional

$$
E_{\Omega}(u):=\int_{\Omega}\left\{\frac{1}{2}|\nabla u|^{2}-G(u)\right\} d x,
$$

where $\Omega \subset \mathbb{R}^{n}, n \geq 2$. Its Euler-Lagrange equation is

$$
-\Delta u=g(u) \quad \text { in } \Omega \text {. }
$$

4.1. Pointwise bounds. We consider radial functions in $H^{1}\left(B_{1}\right)$ that solve the equation in $B_{1} \backslash\{0\}$. That is, we assume

$$
u \in H^{1}\left(B_{1}\right), \quad u=u(r), \quad \text { and }-\Delta u=g(u) \text { in } B_{1} \backslash\{0\} \subset \mathbb{R}^{n} .
$$

Next, we will define the notions of local minimality and semi-stability of $u$. They will concern the energy of $u$, and its second variation, among functions with same boundary values as $u$, that is, equal to $u(1)$ on $\partial B_{1}$. Note that we do not assume $u \geq u(1)$. Hence, in the normalized case when $u(1)=0$, we include solutions that a priori may change sign.

Note that the energy of $u$ in the whole $B_{1}$ is a priori not well defined, since $u$ could be unbounded at the origin and we make no growth assumption on $G$. However, given $\delta>0$, every radial function in $H^{1}\left(B_{1}\right)$ also belongs (as a function of $r=|x|)$ to the Sobolev space $H^{1}(\delta, 1)$ in one dimension. Hence, by the Sobolev embedding in one dimension, away from the origin the function is bounded. This is the reason why the following quantities are well defined.

We say that $u$ is semi-stable if

$$
Q_{u}(\xi):=\int\left\{|\nabla \xi|^{2}-g^{\prime}(u) \xi^{2}\right\} d x \geq 0
$$

for every radial $C^{\infty}$ function $\xi$ with compact support in $B_{1} \backslash\{0\}$. If $u$ is semistable in this sense, then (4.2) also holds for every nonradial $\xi \in C^{\infty}$ with compact support in $B_{1} \backslash\{0\}$. Given such $\xi$, this is proved defining the averages $\xi_{\text {rad }}^{2}(r):=$ $f_{\partial B_{r}} \xi^{2}$, checking that $\left|\partial_{r} \xi_{\text {rad }}(r)\right|^{2} \leq f_{\partial B_{r}}|\nabla \xi|^{2}$, and applying (4.2) to $\xi_{\text {rad }}$.

We say that a radial function $u \in H^{1}\left(B_{1}\right)$ is a radial local minimizer if for every $\delta>0$ there exists $\varepsilon_{\delta}>0$ such that

$$
E_{B_{1} \backslash \bar{B}_{\delta}}(u) \leq E_{B_{1} \backslash \bar{B}_{\delta}}(u+\xi)
$$

for every radial $C^{1}$ function $\xi$ with compact support in $B_{1} \backslash \bar{B}_{\delta}$ and with $\|\xi\|_{C^{1}} \leq$ $\varepsilon_{\delta}$ 
Note that every radial local minimizer $u$ is a semi-stable solution of $-\Delta u=$ $g(u)$ in $B_{1} \backslash\{0\}$. Later in this subsection we will describe another type of semistable solutions, consisting of the smallest (or minimal) solutions of certain problems. They turn out to be one-sided minimizers of the energy and, in particular, semi-stable.

Our main regularity result is the following - more general results are stated in Theorem 4.5 below.

Theorem 4.1 ([5]). Let $g$ be any locally Lipschitz function. Assume that $u \in$ $H^{1}\left(B_{1}\right)$ is a semi-stable radial solution of $-\Delta u=g(u)$ in $B_{1} \backslash\{0\} \subset \mathbb{R}^{n}$. If $n \leq 9$ then $u$ is bounded, and hence regular, in $B_{1}$.

That the dimension 9 is optimal in this result is easily proved with the following example.

Proposition 4.2. For $n \geq 3, U(x)=-2 \log |x|$ is an unbounded radial solution of $-\Delta U=2(n-2) e^{U}$ in $B_{1} \backslash\{0\}$.

$U$ is semi-stable if and only if $n \geq 10$. If $n \geq 11$ then $U$ is in addition a radial local minimizer of the energy with $G(u)=2(n-2) e^{u}$.

In subsection 4.3 we give the proofs of Theorem 4.1 and Proposition 4.2. We will also see that the minimality property of $U$ for $n \geq 11$ holds even in a stronger sense than the one considered in (4.3).

The two previous results were motivated and have applications to the following problems concerning minimal and extremal solutions. We will also explain here some existing versions of Theorem 4.1 for nonradial solutions in general domains.

Consider the problem

$$
\left\{\begin{array}{rlrl}
-\Delta u & =\lambda f(u) & & \text { in } \Omega \\
u \geq 0 & & \text { in } \Omega \\
u & =0 & & \text { on } \partial \Omega,
\end{array}\right.
$$

where $\Omega \subset \mathbb{R}^{n}$ is a smooth bounded domain, $n \geq 2, \lambda \geq 0$, and the nonlinearity $f:[0,+\infty) \rightarrow \mathbb{R}$ satisfies $f$ is $C^{1}$, nondecreasing and convex, $f(0)>0$ and $\lim _{u \rightarrow+\infty} f(u) / u=+\infty$.

It is well known that there exists an extremal parameter $\lambda^{*}$ such that if $0 \leq$ $\lambda<\lambda^{*}$ then $\left(4.4_{\lambda}\right)$ admits a minimal classical solution $u_{\lambda}$. On the other hand, if 
$\lambda>\lambda^{*}$ then $\left(4.4_{\lambda}\right)$ has no classical solution. Here, classical means bounded, while minimal means smallest. The set $\left\{u_{\lambda}: 0 \leq \lambda<\lambda^{*}\right\}$ forms a branch of classical solutions increasing in $\lambda$. Its increasing limit as $\lambda \nearrow \lambda^{*}$ is a solution $u^{*}=u_{\lambda^{*}}$ of $\left(4.4_{\lambda}\right)$ with $\lambda=\lambda^{*}$ in an appropriate weak sense. It is called the extremal solution of $\left(4.4_{\lambda}\right)$.

Every solution $u_{\lambda}$, including the extremal one $u^{*}=u_{\lambda^{*}}$, is in addition semistable. Even more (see [5]), each $u_{\lambda}$ is a one-sided minimizer, in the sense that it is the absolute minimum of the energy in the convex set $\left\{v: 0 \leq v \leq u_{\lambda}\right\}$. Indeed, such a minimizer exists, it is a solution, and hence it must agree with $u_{\lambda}$, since $u_{\lambda}$ is the minimal one.

The extremal solution $u^{*}$ may be regular or singular. When $f(u)=e^{u}$, it is known that $u^{*} \in L^{\infty}(\Omega)$ if $n \leq 9$, for every $\Omega$. This result was proved by Crandall-Rabinowitz [7] and Mignot-Puel [17]. Their proof establishes indeed that Theorem 4.1 also holds in general smooth bounded domains when $f(u)=e^{u}$. On the other hand, we have that $u^{*}(x)=-2 \log |x|$ if $n \geq 10$ and $\Omega=B_{1}$. A similar phenomenon happens when $f(u)=(1+u)^{p}$ with $p>1$.

Brezis and Vázquez [3] raised the question of determining the regularity of $u^{*}$, depending on the dimension $n$, for general nonlinearities $f$ satisfying the conditions above. The best known result is due to Nedev [18], who proved that, for every $\Omega$ and nonlinearity $f$ as above, $u^{*} \in L^{\infty}(\Omega)$ if $n \leq 3$, while $u^{*} \in H_{0}^{1}(\Omega)$ if $n \leq 5$.

Finally, recall that the $L^{\infty}$ bounds of Gidas and Spruck [11] hold for general positive solutions (not necessarily semi-stable, neither radial) but only for nonlinearities of power type with subcritical or critical growth. These estimates are obtained from some Liouville type theorems that we mention in next subsection.

4.2. Semi-stable solutions in the whole space. In this subsection we describe our results in paper [4]. They are concerned with semi-stable radial solutions in the whole space $\mathbb{R}^{n}$, and can also be understood as analogues of the results on minimal cones and minimizing harmonic maps of the previous sections.

Here we assume that $g \in C^{1}(\mathbb{R})$. We say that a bounded solution $u$ of $-\Delta u=$ $g(u)$ in $\mathbb{R}^{n}$ is semi-stable if (4.2) holds for every $C^{\infty}$ function $\xi$ with compact support in $\mathbb{R}^{n}$. This is equivalent to the nonnegativeness of the first eigenvalue of the linearized problem $-\Delta-g^{\prime}(u)$ in every ball. Since here $u$ is a solution in the 
whole space, it follows that all such eigenvalues are indeed positive (see [6]). This is why we used the terminology "stable solution" in [4] —instead of "semi-stable solution" that we use here.

Theorem 4.3 ([4]). Let $u$ be a semi-stable bounded radial solution of $-\Delta u=g(u)$ in $\mathbb{R}^{n}$. If $n \leq 8$ then $u$ is constant.

The same holds for $9 \leq n \leq 10$ if $g$ is analytic or, for instance of one of the forms $g(s)=|s|^{p}, g(s)=|s|^{p-1} s$, for some real exponent $p>1$.

Again, the dimension 10 here is sharp, as the following result shows.

Theorem $4.4([4])$. For $n \geq 11$, the function $u(r)=\left(1+|x|^{2}\right)^{-1 / 8}$ is a nonconstant semi-stable bounded radial solution of

$$
-\Delta u=\left((4 n-9) u^{9}+9 u^{17}\right) / 16=: g(u)
$$

in $\mathbb{R}^{n}$. Note that the nonlinearity $g$ is a polynomial.

See [4] for the proofs of these results, for a more general version of Theorem 4.3 when $9 \leq n \leq 10$, and for some open problems. After submission of this paper, S.Villegas [22] has improved Theorem 4.3 by removing all additional assumptions on $g$ when $9 \leq n \leq 10$.

Theorem 4.3 is a Liouville type theorem for semi-stable radial solutions and for general nonlinearities. For nonlinearities of power type, Liouville type theorems were proved by Gidas and Spruck $[11,12]$ (and later by other authors) for positive solutions not necessarily radial. Recently, Farina [8] has established for power nonlinearities new Liouville theorems for stable solutions - and also for a larger class than that of stable solutions.

4.3. Further estimates and proofs. The following result extends Theorem 4.1. It states sharp regularity results for semi-stable radial solutions. In particular it applies to radial local minimizers.

Theorem 4.5 ([5]). Let $g$ be any locally Lipschitz function. Let $u \in H^{1}\left(B_{1}\right)$ be a semi-stable radial solution of $-\Delta u=g(u)$ in $B_{1} \backslash\{0\}$. Then:

(a) If $n \leq 9$, then $u \in L^{\infty}\left(B_{1}\right)$.

(b) If $n=10$, then $|u(r)| \leq C\|u\|_{H^{1}\left(B_{1}\right)}|\log r|$ for $r<1 / 2$. Here $C$ is a universal constant. 
(c) If $n \geq 11$ and $q<q_{0}:=2 n /(n-2 \sqrt{n-1}-4)$, then $u \in L^{q}\left(B_{1}\right)$. Moreover, for some constant $C_{n}$ depending only on $n$,

$$
|u(r)| \leq C_{n}\|u\|_{H^{1}\left(B_{1}\right)} r^{-n / 2+\sqrt{n-1}+2}|\log r|^{1 / 2} \quad \text { for } r<1 / 2 .
$$

(d) For every $n, u$ is either constant, radially decreasing, or radially increasing in $B_{1}$.

(e) If in addition $g, g^{\prime}$, and $g^{\prime \prime}$ are nonnegative, then $u \in W^{k, q}\left(B_{1}\right)$ for every $k \leq 3$ and $q<q_{k}$. The exponents $q_{k}$ for $k \in\{0,1,2,3\}$ are defined by

$$
\begin{cases}\frac{1}{q_{k}}=\frac{1}{2}-\frac{\sqrt{n-1}}{n}+\frac{k-2}{n} & \text { for } n \geq 10 \\ q_{k}=+\infty & \text { for } n \leq 9 .\end{cases}
$$

A stronger version of the estimates is given in [5], where the $H^{1}$-norm of $u$ in the right hand sides is replaced by its $L^{1}$-norm. This is important for the applications to extremal solutions mentioned in subsection 4.1 .

See also [5] for further estimates and some open problems.

In this subsection we give the proofs of Proposition 4.2 and Theorem 4.5 with the exception of points (d) and (e) of the theorem, that can be found in [5]. In particular, we will have also proved Theorem 4.1.

Proposition 4.2 is quite elementary.

Proof of Proposition 4.2. A simple computation shows that $U$ is a solution in $H^{1}$. To verify the semi-stability (or not) of $U$, we simply compute $g^{\prime}(U)=2(n-2) e^{U}=$ $2(n-2) r^{-2}$ and check that $2(n-2) \leq(n-2)^{2} / 4$ if and only if $n \geq 10$. Recall that $(n-2)^{2} / 4$ is the best constant in Hardy's inequality

$$
\frac{(n-2)^{2}}{4} \int_{B_{1}} \frac{\xi^{2}}{r^{2}} d x \leq \int_{B_{1}}|\nabla \xi|^{2} d x \quad \text { for all } \xi \in C_{c}^{\infty}\left(B_{1}\right)
$$

To prove the local minimality of $U$ when $n \geq 11$, let $\xi \in C_{0}^{1}\left(B_{1}\right)$ satisfy $\|\xi\|_{L^{\infty}} \leq \varepsilon$ for some $\varepsilon$ to be determined later. Then, there exist a function $t$ 
in $B_{1}$ with $|t(x)| \leq 1$ for $x \in B_{1}$, such that

$$
\begin{aligned}
& E_{B_{1}}(U+\xi)=E_{B_{1}}(U)+\frac{1}{2} \int_{B_{1}}\left\{|\nabla \xi|^{2}-g^{\prime}(U) \xi^{2}\right\} d x- \\
& \quad-\int_{B_{1}}\left\{G(U+\xi)-G(U)-g(U) \xi-\frac{1}{2} g^{\prime}(U) \xi^{2}\right\} d x \\
& =E_{B_{1}}(U)+\frac{1}{2} \int_{B_{1}}\left\{|\nabla \xi|^{2}-g^{\prime}(U) \xi^{2}\right\} d x-\frac{1}{6} \int_{B_{1}} g^{\prime \prime}(U+t \xi) \xi^{3} d x
\end{aligned}
$$

Now, using the explicit expressions for $U$ and $g$, we find

$$
\begin{aligned}
& E_{B_{1}}(U+\xi)= \\
& =E_{B_{1}}(U)+\frac{1}{2} \int_{B_{1}}\left\{|\nabla \xi|^{2}-2(n-2) \frac{\xi^{2}}{r^{2}}\right\} d x-\frac{1}{6} \int_{B_{1}} 2(n-2) e^{t \xi} \xi \frac{\xi^{2}}{r^{2}} d x \\
& \geq E_{B_{1}}(U)+\frac{1}{2} \int_{B_{1}}\left\{|\nabla \xi|^{2}-2(n-2) \frac{\xi^{2}}{r^{2}}\right\} d x-\frac{2(n-2) e^{\varepsilon} \varepsilon}{6} \int_{B_{1}} \frac{\xi^{2}}{r^{2}} d x \\
& \geq E_{B_{1}}(U),
\end{aligned}
$$

if we take $\varepsilon$ small enough. The last inequality follows from Hardy's inequality and the fact that $2(n-2)<(n-2)^{2} / 4$ if $n \geq 11$. Hence, we conclude that $U$ is a local minimizer.

To prove Theorem 4.5 we need two preliminary results. The following lemma was inspired by the proof of Theorem 2.1 on minimal cones.

Lemma 4.6 ([5]). Let $u \in H^{1}\left(B_{1}\right)$ be a radial solution of $-\Delta u=g(u)$ in $B_{1} \backslash\{0\}$. Then, for every $\eta \in H^{1}\left(B_{1}\right)$ with compact support in $B_{1} \backslash\{0\}$, we have

$$
Q_{u}\left(r u_{r} \eta\right)=\int_{B_{1}} u_{r}^{2}\left\{|\nabla(r \eta)|^{2}-(n-1) \eta^{2}\right\} d x
$$

where $Q_{u}(\xi)$ is defined by $(4.2)$.

Note that, in contrast with (4.2), expression (4.7) for the quadratic form $Q_{u}$ contains no reference to the nonlinearity $g$. This is the reason why our estimates do not depend on the specific nonlinearity $g$.

We are taking

$$
\xi=c \eta, \quad \text { with } c:=r u_{r},
$$

in the semi-stability property (4.7). Hence, $r u_{r}$ plays here the role of the function $c$ that also appeared when studying minimal cones and harmonic maps. 
The proof of Lemma 4.6 is elementary. It simply uses integration by parts and the equation

$$
-\Delta u_{r}+\frac{n-1}{r^{2}} u_{r}=g^{\prime}(u) u_{r} \quad \text { for } 0<r<1
$$

which is obtained differentiating $-\Delta u=g(u)$ with respect to $r$. Here (4.8) plays the analogue role of the more involved inequalities in Lemmas 2.4 and 3.4.

Using the semi-stability of the solution and Lemma 4.6 we can prove the following result. It is an estimate of the $L^{2}$ norm or $u_{r} r^{-\alpha}$ for certain positive exponents $\alpha$ which depend on the dimension $n$. This estimate is the key ingredient in the proof of Theorem 4.5.

Lemma 4.7 ([5]). Let $n \geq 2$ and $u \in H^{1}\left(B_{1}\right)$ be a semi-stable radial solution of $-\Delta u=g(u)$ in $B_{1} \backslash\{0\}$. Let $\alpha$ satisfy

$$
1 \leq \alpha<1+\sqrt{n-1} .
$$

Then,

$$
\int_{B_{1 / 2}} u_{r}^{2} r^{-2 \alpha} d x \leq \frac{C_{n}}{(n-1)-(\alpha-1)^{2}}\|u\|_{H^{1}\left(B_{1}\right)}^{2},
$$

where $C_{n}$ is a constant depending only on $n$.

Proof. By approximation, the semi-stability of $u$ implies that $Q_{u}(\xi) \geq 0$ for all radial $\xi \in H^{1}\left(B_{1}\right)$ with compact support in $B_{1} \backslash\{0\}$. Hence, Lemma 4.6 leads to

$$
(n-1) \int_{B_{1}} u_{r}^{2} \eta^{2} d x \leq \int_{B_{1}} u_{r}^{2}|\nabla(r \eta)|^{2} d x
$$

for every radial $\eta \in H^{1}\left(B_{1}\right)$ with compact support in $B_{1} \backslash\{0\}$.

Next, using a cut-off function near the origin, one proves that (4.10) also holds for every radial $\eta \in\left(H^{1} \cap L^{\infty}\right)\left(B_{1}\right)$ with compact support in $B_{1}$ (now $\eta$ does not necessarily vanish around 0$)$ and such that $|\nabla(r \eta)| \in L^{\infty}\left(B_{1}\right)$. See [5] for the details of the proof of this. It is here, and only here, where one uses the regularity hypothesis that $u$ belongs to $H^{1}\left(B_{1}\right)$.

Let $\epsilon \in(0,1 / 2)$. For $\alpha \geq 1$ satisfying (4.9), apply (4.10) with $\eta=\eta_{\epsilon}$ given by

$$
\eta_{\epsilon}(r)= \begin{cases}\epsilon^{-\alpha}-2^{\alpha} & \text { if } 0 \leq r \leq \epsilon \\ r^{-\alpha}-2^{\alpha} & \text { if } \epsilon<r \leq 1 / 2 \\ 0 & \text { if } 1 / 2<r .\end{cases}
$$


Note that $\eta_{\epsilon}$ and $\left|\nabla\left(r \eta_{\epsilon}\right)\right|$ are bounded. We obtain

$$
\begin{aligned}
(n-1) \int_{B_{1 / 2} \backslash B_{\epsilon}} & u_{r}^{2}\left(r^{-\alpha}-2^{\alpha}\right)^{2} d x+(n-1)\left(\epsilon^{-\alpha}-2^{\alpha}\right)^{2} \int_{B_{\epsilon}} u_{r}^{2} d x \\
\leq & \int_{B_{1 / 2} \backslash B_{\epsilon}} u_{r}^{2}\left((1-\alpha) r^{-\alpha}-2^{\alpha}\right)^{2} d x+\left(\epsilon^{-\alpha}-2^{\alpha}\right)^{2} \int_{B_{\epsilon}} u_{r}^{2} d x .
\end{aligned}
$$

Since $n \geq 2$, it follows that

$$
(n-1) \int_{B_{1 / 2} \backslash B_{\epsilon}} u_{r}^{2}\left(r^{-\alpha}-2^{\alpha}\right)^{2} d x \leq \int_{B_{1 / 2} \backslash B_{\epsilon}} u_{r}^{2}\left((1-\alpha) r^{-\alpha}-2^{\alpha}\right)^{2} d x .
$$

Developing the squares, using $n \geq 2$ and (4.9), we find the estimate

$$
\int_{B_{1 / 2} \backslash B_{\epsilon}} u_{r}^{2} r^{-2 \alpha} d x \leq \frac{C_{n}}{(n-1)-(\alpha-1)^{2}} \int_{B_{1 / 2} \backslash B_{\epsilon}} u_{r}^{2} r^{-\alpha} d x .
$$

Throughout the proof, $C_{n}$ (respectively, $C_{\alpha, n}$ ) denote different positive constants depending only on $n$ (respectively, on $\alpha$ and $n$ ).

Now, choose a positive constant $C_{\alpha, n}$ such that

$$
\frac{C_{n}}{(n-1)-(\alpha-1)^{2}} r^{-\alpha} \leq \frac{1}{2} r^{-2 \alpha}+C_{\alpha, n} \quad \text { for all } r>0
$$

The previous inequality and (4.11) lead to

$$
\int_{B_{1 / 2} \backslash B_{\epsilon}} u_{r}^{2} r^{-2 \alpha} d x \leq C_{\alpha, n}\|u\|_{H^{1}\left(B_{1}\right)}^{2} .
$$

Note that we want to have a precise expression, depending on $\alpha$, of the previous constant $C_{\alpha, n}$. To obtain it, we apply (4.12) with the special choice $\alpha=(1+\sqrt{n-1}) / 2 \in[1,1+\sqrt{n-1})$ to deduce

$$
\int_{B_{1 / 2}} u_{r}^{2} r^{-(1+\sqrt{n-1})} d x \leq C_{n}\|u\|_{H^{1}\left(B_{1}\right)}^{2}
$$

Finally, coming back to our general $\alpha$, since $r^{-\alpha} \leq r^{-(1+\sqrt{n-1})}$ in $B_{1},(4.11)$ and (4.13) lead to the desired estimate of Lemma 4.7 after letting $\varepsilon \rightarrow 0$.

We can finally give the proof of the main estimates. 
Proof of Theorem 4.5(a),(b),(c). Let $\alpha$ satisfy (4.9). For $0<s \leq 1 / 2$, we have

$$
\begin{aligned}
|u(s)-u(1 / 2)| & =\left|\int_{s}^{1 / 2}-u_{r} d r\right| \leq \int_{s}^{1 / 2}\left|u_{r}\right| r^{-\alpha+\frac{n-1}{2}} r^{\alpha-\frac{n-1}{2}} d r \\
& \leq C_{n}\left(\int_{B_{1 / 2}} u_{r}^{2} r^{-2 \alpha} d x\right)^{1 / 2}\left(\int_{s}^{1 / 2} r^{2 \alpha+1-n} d r\right)^{1 / 2}
\end{aligned}
$$

by Cauchy-Schwarz. Using Lemma 4.7, we deduce

$$
|u(s)-u(1 / 2)| \leq \frac{C_{n}}{\sqrt{(n-1)-(\alpha-1)^{2}}}\left(\int_{s}^{1 / 2} r^{2 \alpha+1-n} d r\right)^{1 / 2}\|u\|_{H^{1}\left(B_{1}\right)}
$$

for all $0<s<1 / 2$.

(a) Assume $n \leq 9$. The integral in (4.15) is finite with $s=0$ if we take $2 \alpha+1-n>-1$, i.e.,

$$
(n-4) / 2<\alpha-1 .
$$

Since $n \leq 9$, then $(n-4) / 2<\sqrt{n-1}$ and we can choose $\alpha$ satisfying (4.16) and $\alpha<1+\sqrt{n-1}$, so that Lemma 4.7 holds. Note also that $|u(1 / 2)|$ can be controlled by $\|u\|_{H^{1}}$ using the Sobolev embedding in one dimension. Now, the desired estimate follows from (4.15).

(b) Assume $n=10$. For $0<\varepsilon<1$, let $\alpha=4-\varepsilon$ and apply Lemma 4.7 to obtain

$$
\int_{B_{1 / 2}} u_{r}^{2} r^{-8+2 \varepsilon} d x \leq \frac{C}{\varepsilon}\|u\|_{H^{1}\left(B_{1}\right)}^{2}
$$

for a universal constant $C$ (independent of $\varepsilon$ ). This estimate and (4.14) give

$$
\begin{aligned}
|u(s)-u(1 / 2)| & \leq \frac{C}{\sqrt{\varepsilon}}\left(\int_{s}^{1 / 2} r^{-1-2 \varepsilon} d r\right)^{1 / 2}\|u\|_{H^{1}\left(B_{1}\right)} \\
& \leq C\|u\|_{H^{1}\left(B_{1}\right)} \frac{s^{-\varepsilon}}{\varepsilon}
\end{aligned}
$$

for $0<s<1 / 2$ and every $0<\varepsilon<1$. From this, it follows that $u \in L^{q}\left(B_{1}\right)$ for every $q<\infty$.

In order to prove the pointwise estimate logarithmic bound, given $s \in(0,1 / 2)$ we find the $\varepsilon$ that minimizes $s^{-\varepsilon} / \varepsilon$ in (4.17). We obtain

$$
\varepsilon=|\log s|^{-1} \text {. }
$$


Note that $\varepsilon \in(0,1)$ if $s<e^{-1}$. Finally, using (4.17) with $\varepsilon$ given by (4.18), we obtain

$$
|u(s)-u(1 / 2)| \leq C\|u\|_{H^{1}\left(B_{1}\right)}|\log s| \quad \text { for } 0<s<e^{-1} .
$$

From this the desired logarithmic bound follows.

(c) Assume $n \geq 11$ and let $\varepsilon \in(0,1)$. Using (4.15) we argue as part (b) with $\alpha=1+\sqrt{n-1}-\varepsilon$ to obtain

$$
|u(s)-u(1 / 2)| \leq \frac{C_{n}}{\sqrt{\varepsilon}}\|u\|_{H^{1}\left(B_{1}\right)} s^{-n / 2+\sqrt{n-1}+2-\varepsilon}
$$

for $0<s<1 / 2$, where we have used that $-n+2 \sqrt{n-1}+4<0$ since $n \geq 11$.

Now, we use (4.19) to calculate, for $q \geq 1$,

$$
\int_{B_{1 / 2}}|u-u(1 / 2)|^{q} d x \leq\|u\|_{H^{1}\left(B_{1}\right)}^{q} \frac{C_{n}^{q}}{\varepsilon^{q / 2}} \int_{0}^{1 / 2} s^{n-1+q(-n / 2+\sqrt{n-1}+2-\varepsilon)} d s .
$$

If we set $q=2 n /(n-2 \sqrt{n-1}-4+3 \varepsilon)$, then the second integral in (4.20) is finite for every $\varepsilon>0$ small enough. Hence, $u \in L^{q}\left(B_{1 / 2}\right)$ for every $q<q_{0}:=$ $2 n /(n-2 \sqrt{n-1}-4)$ and the estimate follows.

To prove the pointwise estimate (4.5), we proceed as in part (b) by choosing $\varepsilon=-1 /(2 \log s)$.

4.4. The p-Laplacian. Finally, we mention a further result regarding semistable radial solutions to reaction problems involving the $p$-Laplacian.

The methods presented to prove Theorem 4.5 can be adapted to the case of an energy functional of the form

$$
E_{\Omega}(u):=\int_{\Omega}\left\{\frac{1}{p}|\nabla u|^{p}-G(u)\right\} d x
$$

where $1<p<\infty$. In this case we can also prove sharp pointwise, $L^{q}$ and, for $g=G^{\prime}$ nonnegative, $W^{1, q}$ estimates for semi-stable radially decreasing solutions $u$ of the Euler-Lagrange problem:

$$
\left\{\begin{aligned}
-\operatorname{div}\left(|\nabla u|^{p-2} \nabla u\right) & =g(u) & & \text { in } B_{1} \\
u & >0 & & \text { in } B_{1} \\
u & =0 & & \text { on } \partial B_{1} .
\end{aligned}\right.
$$

We include all locally Lipschitz nonlinearities $g:[0,+\infty) \rightarrow \mathbb{R}$ and exponents $p>1$. For this problem, we also define sharp exponents $q_{k}(p)$ for $k \in\{0,1\}$ and 
$p>1$ that extend the ones in (4.6) for $p=2$. For further details see [6] and a forthcoming paper.

\section{ACKNowledgments}

Both authors were supported by the Ministerio de Educación y Ciencia (Spain), grant MTM2005-07660-C02-01. The authors are partially sponsored by an E.U. grant through the RTN Program "Front-Singularities" HPRN-CT-2002-00274, and by the European Science Foundation (ESF) PESC Programme "Global".

The second author is also supported by the E.U. RTN program "MULTIMAT" MRTN-CT-2004-505226.

\section{REFERENCES}

[1] Alberti, G., Ambrosio, L., Cabré, X. On a long-standing conjecture of E. De Giorgi: symmetry in $3 D$ for general nonlinearities and a local minimality property, Acta Appl. Math. 65, 2001, 9-33.

[2] Bombieri, E., De Giorgi, E., Giusti, E. Minimal cones and the Bernstein problem, Inv. Math. 7, 1969, 243-268.

[3] Brezis, H., Vázquez, J.L. Blow-up solutions of some nonlinear elliptic problems, Rev. Mat. Univ. Compl. Madrid 10, 1997, 443-469.

[4] Cabré, X., Capella, A. On the stability of radial solutions of semilinear elliptic equations in all of $\mathbb{R}^{n}$, C. R. Math. Acad. Sci. Paris 338, 2004, 769-774.

[5] Cabré, X., Capella, A. Regularity of radial minimizers and extremal solutions of semilinear elliptic equations, J. of Functional Analysis 238, 2006, 709-733.

[6] Capella, A. Stable solutions of nonlinear elliptic equations: qualitative and regularity properties, Doctoral Thesis, Universitat Politècnica de Catalunya, 2005.

[7] Crandall, M.G., Rabinowitz, P.H. Some continuation and variational methods for positive solutions of nonlinear elliptic eigenvalue problems, Arch. Rat. Mech. Anal. 58, 1975, 207218.

[8] Farina, A. Liouville-type results for solutions of $-\Delta u=|u|^{p-1} u$ on unbounded domains of $\mathbb{R}^{N}$, C. R. Math. Acad. Sci. Paris 341, 2005, 415-418.

[9] Giaquinta, M. Introduction to regularity theory for nonlinear elliptic systems, Lectures in Mathematics ETH Zürich. Birkhäuser Verlag, Basel, 1993.

[10] Giaquinta, M., Souček, J. Harmonic maps into a hemisphere, Ann. Scuola Norm. Sup. Pisa Cl. Sci. (4) 12, 1985, 81-90.

[11] Gidas, B., Spruck, J. A priori bounds for positive solutions of nonlinear elliptic equations, Comm. Partial Differential Equations 6, 1981, 883-901.

[12] Gidas, B., Spruck, J. Global and local behavior of positive solutions of nonlinear elliptic equations, Comm. Pure Appl. Math. 34, 1981, 525-598. 
[13] Giusti, E. Minimal Surfaces and Functions of Bounded Variation, Monographs in Mathematics 80, Birkhäuser Verlag, Basel, 1984.

[14] Hardt, R.M. Singularities of harmonic maps, Bull. Amer. Math. Soc. (N.S.) 34, 1997, $15-34$.

[15] Jäger, W., Kaul, H. Rotationally symmetric harmonic maps from a ball into a sphere and the regularity problem for weak solutions of elliptic systems, J. Reine Angew. Math. 343, 1983, 146-161.

[16] Jerison, D., Monneau, R. Towards a counter-example to a conjecture of De Giorgi in high dimensions, Ann. Mat. Pura Appl. 183, 2004, 439-467.

[17] Mignot, F., Puel, J.-P. Sur une classe de problèmes non linéaires avec nonlinéarité positive, croissante, convexe, Comm. Partial Differential Equations 5, 1980, 791-836.

[18] Nedev, G. Regularity of the extremal solution of semilinear elliptic equations, C. R. Acad. Sci. Paris, Série I 330, 2000, 997-1002.

[19] Savin, O. Phase transitions: Regularity of flat level sets, preprint.

[20] Schoen, R., Uhlenbeck, K. Regularity of minimizing harmonic maps into the sphere, Invent. Math. 78, 1984, 89-100.

[21] Simons, J. Minimal varieties in riemannian manifolds, Ann. of Math. 88, 1968, 62-105.

[22] Villegas, S. Asymptotic behavior of stable radial solutions of semilinear elliptic equations in $\mathbb{R}^{N}$, preprint.

Xavier Cabré

ICREA and Universitat Politècnica de Catalunya

Departament de Matemàtica Aplicada I

Diagonal 647, 08028 Barcelona, Spain

E-mail: xavier.cabre@upc.edu

Antonio Capella

Institut für Angewandte Mathematik der Universität Bonn

Abteilung für Mathematische Methoden der Physik

Wegelerstr. 10, 53115 Bonn, Germany

E-mail: capella@iam.uni-bonn.de 